\title{
Pseudo-Predictor Feedback for Neutral-Type Linear Systems with Both State and Input Delays*
}

\author{
Bin Zhou ${ }^{\dagger} \quad$ Qingsong Liu ${ }^{\dagger} \quad$ Wim Michiels ${ }^{\ddagger}$
}

\begin{abstract}
In this paper, the stabilization problem of neutral-type linear time-delay systems with both state and input delays is studied. A pseudo-predictor feedback (PPF) approach is established to predict the future states such that the input delay is compensated properly. It is shown that the considered system can be stabilized by the PPF controller if and only if an associated integral delay system is asymptotically stable. Moreover, it is proved that the PPF controller can be safely implemented without involving any input filters. The computing the feedback gains problem is successfully converted to a simultaneous stabilization problem, which is solved by adopting a direct optimization approach. The proposed PPF approach is also extended to neutral-type time-varying time-delay systems. A numerical example is carried out to illustrate the effectiveness of the proposed controllers.
\end{abstract}

Keywords: Neutral-type time-delay systems; Pseudo-predictor feedback; State and input delays; Stabilization.

\section{Introduction}

Analysis and design of time-delay systems are attractive topics on which many significant results have been reported in the past several decades (see [5, 26, 32] and the references therein). Stabilization is one of the fundamental design problems for time-delay systems, and has thus been extensively studied in the literature. For example, the stabilization problem of interconnected systems with input delays was addressed in [13], the stabilization of linear neutral-type functional differential equations was presented in [18] by using an eigenvalue based approach, the stabilization of linear strict-feedback systems with delayed integrators was considered in [2], and the stabilization of linear systems with input, state and output delays was investigated in [4] by chains of predictors. It should be pointed out that, unlike retarded-type time-delay systems, neutral-type time-delay systems have received much less attention, whereas they can be used to model many engineering systems such as lossless transmission lines [11], partial element equivalent circuits [1], combustion systems [17] and controlled constrained manipulators in mechanical engineering [23].

The predictor feedback approach has been already widely applied to the control of time-delay systems, and there are a large number of results reported in the literature that deal with various analysis and design problems. For instance, the Smith predictor was firstly proposed in [27] to compensate the input delay such that the closed-loop system turns into a delay-free system, a modified Smith predictor was later suggested in [29] since the Smith predictor may fail to stabilize open-loop unstable systems, an alternative scheme for delay elimination from the closed-loop referred to as the finite spectrum assignment strategy was studied comprehensively in [30], the exponential stability of linear systems with distributed actuator delays and predictor feedback was investigated by the use of Lyapunov functionals in [3], and the stabilization problem for nonlinear systems with input delays was studied in [8] by means of approximate predictors. Recently, for linear systems with both state and input delays, a novel method referred to as nested predictor feedback was

\footnotetext{
* This work was supported in part by the National Natural Science Foundation of China under grant number 61773140, in part by the KU Leuven Research Council under grant number C14/17/072, and in part by the Research Foundation-Flanders (FWO - Vlaanderen) under grant number G0A5317N.

${ }^{\dagger}$ Bin Zhou and Qingsong Liu are with the Center for Control Theory and Guidance Technology, Harbin Institute of Technology, Harbin, 150001, China. Email: binzhoulee@163.com, binzhou@hit.edu.cn.

${ }^{\ddagger}$ W. Michiels is with the Department of Computer Science, KU Leuven, Celestijnenlaan 200A, 3001 Leuven, Belgium. Email: wim.michiels@cs.kuleuven.be.
} 
proposed in [36] to study the long input delay compensation problem. This method was later extended to discrete-time time-delay systems [14], neutral-type time-delay systems [35], and to the case of linear systems with both state and distinct input delays [15]. Almost at the same time, the same problem was independently investigated in [9] by a completely different method which is based on the fundamental matrix functions for open-loop time-delay systems. The approach proposed in [9] was also extended to neutral-type time-delay systems [10].

Recently, a new method referred to as pseudo-predictor feedback (PPF) was proposed in [33] to study the stabilization of linear systems with only (time-varying) input delays. Different from the traditional predictor feedback, the PPF results in controllers that are finite dimensional and are thus very easy to implement. The PPF approach proposed in [33] was later extended to linear systems with both pointwise and distributed input delays [16]. In this paper, motivated by the PPF approach proposed in [33] for retarded-type linear systems with only an input delay, we use the PPF approach to study the stabilization problem for neutraltype linear systems with both state and input delays. We will show the PPF approach proposed in [33] for retarded-type linear systems with only an input delay can be successfully extended to the case of neutraltype systems with both state and input delays. We point out that the extension of the PPF to neutral-type time-delay systems with both state and input delays is nontrivial. For example, the PPF controller for linear systems with only an input delay is finite dimensional, while the PPF controller for linear system with both input and state delays is still infinite dimensional. However, compared with the traditional predictor feedback which may suffer some implementation problems, we will show that the PPF controller can be safely implemented via numerical integrations without using any input filters. We will show that the computation of the feedback gains problem can be converted to a simultaneous stabilization problem, which is solved by adopting a direct optimization approach. In addition, it is shown that the proposed PPF approach can be extended to neutral-type time-varying time-delay systems. The effectiveness of the proposed approach will be illustrated by a numerical example.

This paper is organized as follows: In Section 2, we give some preliminaries and the problem formulation. The design of the PPF, the stability analysis of the closed-lop system, and the computation of the feedback gains problem is addressed in Section 3. The proposed PPF approach is then extended to time-varying timedelay systems in Section ??. In Section ??, an example is worked out to support the obtained theoretical results. Finally, Section ?? concludes this paper.

\section{Preliminaries and Problem Formulation}

We consider the following neutral-type time-delay system with both state and input delays

$$
\frac{\mathrm{d}}{\mathrm{d} t}[x(t)-D x(t-r)]=A_{0} x(t)+A_{1} x(t-r)+B u(t-r),
$$

where $A_{0}, A_{1} \in \mathbf{R}^{n \times n}$ and $B \in \mathbf{R}^{n \times m}$ are constant matrices, $D \in \mathbf{R}^{n \times n}$ is assumed to be Schur stable, and $r>0$ is the state/input delay. In this paper, we are interested in designing stabilizing controllers for the neutral-type time-delay system (1) with both input and state delays. The problem can be stated formally as follows.

Problem 1 Design for the neutral-type linear time-delay system (1) a linear controller

$$
u(t)=F\left(x_{t}\right),
$$

such that the closed-loop system is asymptotically stable, where $F$ is some linear function.

We study this problem under the following assumption.

Assumption 1 There are feedback gains $K_{i} \in \mathbf{R}^{m \times n}, i=1,2$, such that the following neutral-time timedelay system is asymptotically stable

$$
\frac{\mathrm{d}}{\mathrm{d} t}[x(t)-D x(t-r)]=\left(A_{0}+B K_{0}\right) x(t)+\left(A_{1}+B K_{1}\right) x(t-r),
$$


We point out that the determine of the feedback gains $K_{0}$ and $K_{1}$ in (2) has been studied in our early work (see Lemma 1 in [35]) and thus will not be investigated here. The reason behind Assumption 1 is that system (1) in the absence of the input delay can be stabilized by the linear feedback

$$
u(t)=K_{0} x(t)+K_{1} x(t-r) .
$$

Therefore, Problem 1 is equivalent to finding an appropriate way to compensate the input time delay.

For linear systems with both state and input delays, we know that the model reduction approach can deal with the stabilization problem, which however is not as simple as the case without state delays since some nonlinear matrix equations in terms of the coefficient matrices of the system are needed to be solved [6, 25]. Recently, Problem 1 was studied in [10] by using the fundamental matrix functions associated with the open-loop time-delay system, and was also considered in [36] by the nested predictor feedback. However, the resulting controllers in both [10] and [36] will involve integral terms such as

$$
\int_{-r}^{0} \mathrm{e}^{-A_{0} s} B u(t+s) \mathrm{d} s
$$

which needs to be implemented by numerical integrations. The possible drawback of the numerical integration is that the closed-loop system may not be asymptotically stable no matter how precise the numerical integration is [28]. The implementation problem in [10] and [36] can be overcome by adding some low pass input filters, which however might be expensive in practice.

Pseudo-predictor feedback (PPF) was recently proposed in [33] to study the stabilization problem of retardedtype linear system with only input delays. Differently from the traditional predictor feedback which uses the open-loop system dynamics to predict the future state and will result in a memory controller, the PPF uses the (artificial) closed-loop system dynamics to predict the future state. As a result, the obtained controller is memoryless and is thus easy to implement. In this paper, the PPF approach will be adopted to solve the stabilization problem for neutral-type linear systems with both state and input delays. It is shown that computing the feedback gains problem can be converted to a simultaneous stabilization problem that is solved by using a direct optimization approach (see Subsection 3.3 for details). It is also proved that the PPF controller established in this paper can be safely implemented without using any input filters even in the presence of integral terms. Moreover, the proposed PPF approach can be extended to neutral-type time-varying time-delay systems (see Section ?? for details).

\section{Pseudo-Predictor Feedback}

\subsection{The Design of Pseudo-Predictor Feedback}

Notice that system (1) with the following controller

$$
u(t)=K_{0} x(t+r)+K_{1} x(t),
$$

will possess the same characteristic polynomial as system (2) which will be asymptotically stable by Assumption 1. As the controller (4) involves the future state $x(t+r)$, we need to use certain system model to predict it. Let

$$
\varphi(t)=x(t)-D x(t-r) .
$$

Then the open-loop system (1) can be expressed by

$$
\dot{\varphi}(t)=A_{0} \varphi(t)+\left(A_{0} D+A_{1}\right) x(t-r)+B u(t-r),
$$

from which it follows that (by the variation of constant formula)

$$
\varphi(t+r)=\mathrm{e}^{A_{0} r} \varphi(t)+\int_{t}^{t+r} \mathrm{e}^{A_{0}(t+r-s)}\left(\left(A_{0} D+A_{1}\right) x(s-r)+B u(s-r)\right) \mathrm{d} s,
$$

or, equivalently,

$$
x(t+r)=D x(t)+\mathrm{e}^{A_{0} r}(x(t)-D x(t-r))+\int_{t}^{t+r} \mathrm{e}^{A_{0}(t+r-s)}\left(\left(A_{0} D+A_{1}\right) x(s-r)+B u(s-r)\right) \mathrm{d} s
$$




$$
=\left(\mathrm{e}^{A_{0} r}+D\right) x(t)-\mathrm{e}^{A_{0} r} D x(t-r)+\int_{t-r}^{t} \mathrm{e}^{A_{0}(t-s)}\left(\left(A_{0} D+A_{1}\right) x(s)+B u(s)\right) \mathrm{d} s .
$$

Substituting the above expression into (4) gives the following conventional predictor feedback

$$
\begin{aligned}
u(t)= & \left(K_{0}\left(\mathrm{e}^{A_{0} r}+D\right)+K_{1}\right) x(t)-K_{0} \mathrm{e}^{A_{0} r} D x(t-r) \\
& +K_{0} \int_{t-r}^{t} \mathrm{e}^{A_{0}(t-s)}\left(\left(A_{0} D+A_{1}\right) x(s)+B u(s)\right) \mathrm{d} s .
\end{aligned}
$$

It can be shown that the closed-loop system consisting of (1) and (7) possess the same characteristic equation as system (2), and is thus asymptotically stable by Assumption 1.

The conventional predictor feedback (7) was obtained by using the open-loop system dynamics (1) to predict $x(t+h)$. As the usual predictor feedback for retarded-type time-delay systems, the resulting controller (7) is infinite dimensional because of terms like $\int_{-h}^{0} \mathrm{e}^{-A_{0} s} B u(t+s) \mathrm{d} s$, which should be implemented by numerical integration. However, such an approximated implementation may lead to some instability problems, namely, the closed-loop system may not be asymptotically stable no matter how precise the numerical integration is $[28]$.

Remark 1 We point out that, when implementing stabilizing conventional predictor feedback (7) by discretizing the integral by a quadrature rule, a necessary condition for preservation of stability is that the following integral delay system (IDS)

$$
u(t)=K_{0} \int_{t-r}^{t} \mathrm{e}^{A_{0}(t-s)} B u(s) \mathrm{d} s,
$$

is asymptotically stable. In fact, it is necessary and sufficient if there is no perturbation on the delays in the discretized distribution delay [19].

Motivated by the first author's early study [33], we will establish a PPF instead of the conventional predictor feedback to predict $x(t+r)$. The idea is to use the virtual closed-loop system dynamics (2) instead of the open-loop system (1) to predict $x(t+r)$. By using (6), where $A_{0}$ is replaced by $A_{0}+B K_{0}, A_{1}$ is replaced by $A_{1}+B K_{1}$, and $u=0$, we obtain

$$
\begin{aligned}
x(t+r)= & \left(\mathrm{e}^{\left(A_{0}+B K_{0}\right) r}+D\right) x(t)-\mathrm{e}^{\left(A_{0}+B K_{0}\right) r} D x(t-r) \\
& +\int_{t-r}^{t} \mathrm{e}^{\left(A_{0}+B K_{0}\right)(t-s)}\left(\left(A_{0}+B K_{0}\right) D+A_{1}+B K_{1}\right) x(s) \mathrm{d} s .
\end{aligned}
$$

The PPF controller can then be obtained by substituting (9) into (4) as

$$
\begin{aligned}
u(t)= & K_{0} x(t+r)+K_{1} x(t) \\
= & \left(K_{0}\left(\mathrm{e}^{\left(A_{0}+B K_{0}\right) r}+D\right)+K_{1}\right) x(t)-K_{0} \mathrm{e}^{\left(A_{0}+B K_{0}\right) r} D x(t-r) \\
& +K_{0} \int_{t-r}^{t} \mathrm{e}^{\left(A_{0}+B K_{0}\right)(t-s)}\left(\left(A_{0}+B K_{0}\right) D+A_{1}+B K_{1}\right) x(s) \mathrm{d} s .
\end{aligned}
$$

The name "pseudo-predictor feedback" follows from the fact that the closed-loop system (2) does not exist actually.

\subsection{Stability Analysis of the Closed-Loop System}

The closed-loop system consisting of system (1) and the PPF (10) is given by

$$
\begin{aligned}
\frac{\mathrm{d}}{\mathrm{d} t}[x(t)-D x(t-r)]= & A_{0} x(t)+A_{1} x(t-r) \\
& +B\left(\left(K_{0}\left(\mathrm{e}^{\left(A_{0}+B K_{0}\right) r}+D\right)+K_{1}\right) x(t-r)-K_{0} \mathrm{e}^{\left(A_{0}+B K_{0}\right) r} D x(t-2 r)\right.
\end{aligned}
$$




$$
\left.+K_{0} \int_{t-2 r}^{t-r} \mathrm{e}^{\left(A_{0}+B K_{0}\right)(t-r-s)}\left(\left(A_{0}+B K_{0}\right) D+A_{1}+B K_{1}\right) x(s) \mathrm{d} s\right),
$$

whose stability should be carefully checked. The following theorem provides necessary and sufficient conditions for the stability of the above closed-loop system.

Theorem 1 Let Assumption 1 be satisfied. Then the closed-loop system (11) is asymptotically stable if and only if the following integral delay system (IDS) is asymptotically stable

$$
\rho(t)=-K_{0} \int_{t-r}^{t} \mathrm{e}^{\left(A_{0}+B K_{0}\right)(t-s)} B \rho(s) \mathrm{d} s
$$

Proof. Let $\Delta_{1}(s)$ and $\Delta_{2}(s)$ be the characteristic equations for the system (2) and the IDS (12), respectively, namely,

$$
\Delta_{1}(s)=\left|s I_{n}-s D \mathrm{e}^{-r s}-\left(A_{0}+B K_{0}\right)-\left(A_{1}+B K_{1}\right) \mathrm{e}^{-r s}\right|,
$$

and

$$
\Delta_{2}(s)=\left|I_{m}+K_{0}\left(s I_{n}-A_{0}-B K_{0}\right)^{-1}\left(I_{n}-\mathrm{e}^{-\left(s I_{n}-A_{0}-B K_{0}\right) r}\right) B\right| .
$$

Notice that the characteristic equation of the closed-loop system (11) is given by

$$
\begin{aligned}
\Delta(s)= & \operatorname{det}\left(s I_{n}-s D \mathrm{e}^{-r s}-A_{0}-\left(A_{1}+B\left(K_{0} D+K_{1}\right)\right) \mathrm{e}^{-r s}-B K_{0} \mathrm{e}^{-\left(s I_{n}-A_{0}-B K_{0}\right) r}\left(I_{n}-D \mathrm{e}^{-r s}\right)\right. \\
& \left.-B K_{0}\left(s I_{n}-A_{0}-B K_{0}\right)^{-1}\left(I_{n}-\mathrm{e}^{-\left(s I_{n}-A_{0}-B K_{0}\right) r}\right)\left(\left(A_{0}+B K_{0}\right) D+A_{1}+B K_{1}\right) \mathrm{e}^{-r s}\right) .
\end{aligned}
$$

It follows that

$$
\begin{aligned}
\Delta(s)= & \operatorname{det}\left(\Delta_{1}(s)+B K_{0}-B K_{0} D \mathrm{e}^{-r s}-B K_{0} \mathrm{e}^{-\left(s I_{n}-A_{0}-B K_{0}\right) r}\left(I_{n}-D \mathrm{e}^{-r s}\right)\right. \\
& \left.-B K_{0}\left(s I_{n}-A_{0}-B K_{0}\right)^{-1}\left(I_{n}-\mathrm{e}^{-\left(s I_{n}-A_{0}-B K_{0}\right) r}\right)\left(\left(A_{0}+B K_{0}\right) D+A_{1}+B K_{1}\right) \mathrm{e}^{-r s}\right) \\
= & \operatorname{det}\left(s I_{n}-s D \mathrm{e}^{-r s}-\left(A_{0}+B K_{0}\right)-\left(A_{1}+B K_{1}\right) \mathrm{e}^{-r s}\right. \\
& +B K_{0}\left(I_{n}-\mathrm{e}^{-\left(s I_{n}-A_{0}-B K_{0}\right) r}\right)-B K_{0}\left(I_{n}-\mathrm{e}^{-\left(s I_{n}-A_{0}-B K_{0}\right) r}\right) D \mathrm{e}^{-r s} \\
& \left.-B K_{0}\left(s I_{n}-A_{0}-B K_{0}\right)^{-1}\left(I_{n}-\mathrm{e}^{-\left(s I_{n}-A_{0}-B K_{0}\right) r}\right)\left(\left(A_{0}+B K_{0}\right) D+A_{1}+B K_{1}\right) \mathrm{e}^{-r s}\right) \\
= & \operatorname{det}\left(s I_{n}-s D \mathrm{e}^{-r s}-\left(A_{0}+B K_{0}\right)-\left(A_{1}+B K_{1}\right) \mathrm{e}^{-r s}\right. \\
& +B K_{0}\left(s I_{n}-A_{0}-B K_{0}\right)^{-1}\left(I_{n}-\mathrm{e}^{-\left(s I_{n}-A_{0}-B K_{0}\right) r}\right)\left(s I_{n}-A_{0}-B K_{0}\right) \\
& -B K_{0}\left(s I_{n}-A_{0}-B K_{0}\right)^{-1}\left(I_{n}-\mathrm{e}^{-\left(s I_{n}-A_{0}-B K_{0}\right) r}\right)\left(s I_{n}-A_{0}-B K_{0}\right) D \mathrm{e}^{-r s} \\
& \left.-B K_{0}\left(s I_{n}-A_{0}-B K_{0}\right)^{-1}\left(I_{n}-\mathrm{e}^{-\left(s I_{n}-A_{0}-B K_{0}\right) r}\right)\left(\left(A_{0}+B K_{0}\right) D+A_{1}+B K_{1}\right) \mathrm{e}^{-r s}\right) \\
= & \operatorname{det}\left(s I_{n}-s D \mathrm{e}^{-r s}-\left(A_{0}+B K_{0}\right)-\left(A_{1}+B K_{1}\right) \mathrm{e}^{-r s}\right. \\
& +B K_{0}\left(s I_{n}-A_{0}-B K_{0}\right)^{-1}\left(I_{n}-\mathrm{e}^{-\left(s I_{n}-A_{0}-B K_{0}\right) r}\right)\left(s I_{n}-A_{0}-B K_{0}\right. \\
& \left.\left.-\left(s I_{n}-A_{0}-B K_{0}\right) D \mathrm{e}^{-r s}-\left(\left(A_{0}+B K_{0}\right) D+A_{1}+B K_{1}\right) \mathrm{e}^{-r s}\right)\right) \\
= & \operatorname{det}\left(I_{n}+B K_{0}\left(s I_{n}-A_{0}-B K_{0}\right)^{-1}\left(I_{n}-\mathrm{e}^{-\left(s I_{n}-A_{0}-B K_{0}\right) r}\right)\right) \Delta_{1}(s) \\
= & \operatorname{det}\left(I_{m}+K_{0}\left(s I_{n}-A_{0}-B K_{0}\right)^{-1}\left(I_{n}-\mathrm{e}^{-\left(s I_{n}-A_{0}-B K_{0}\right) r}\right) B\right) \Delta_{1}(s) \\
= & \Delta_{2}(s) \Delta_{1}(s),
\end{aligned}
$$

where we have used $\operatorname{det}(I+C L)=\operatorname{det}(I+L C)$ with $C$ and $L$ having appropriate dimensions. This allows us to conclude.

We mention that the IDS (12) coincides exactly with the IDS (17) in [33], where sufficient conditions guaranteeing the stability of such an IDS have been provided, and thus will not be recalled here. In addition, it is interesting to notice that the IDS (12) is independent of $A_{1}, K_{1}$ and $D$. With this observation, we can present the following result. 
Corollary 1 Assume that $A_{0}+B K_{0}$ is Hurwitz. Then the IDS (12) is asymptotically stable if and only if the following retarded-type time-delay system is asymptotically stable

$$
\dot{y}(t)=A_{0} y(t)+\mathrm{e}^{\left(A_{0}+B K_{0}\right) r} B K_{0} y(t-r) .
$$

Proof. Let $\lambda_{\mathrm{ID}}$ and $\lambda_{\mathrm{D}}$ be the characteristic eigenvalue sets for the delay system (12) and (13), respectively, namely,

$$
\lambda_{\mathrm{ID}}=\left\{s: \operatorname{det}\left(I_{m}+\int_{-r}^{0} K_{0} \mathrm{e}^{-\left(A_{0}+B K_{0}\right) \theta} B \mathrm{e}^{s \theta} \mathrm{d} \theta\right)=0\right\},
$$

and

$$
\lambda_{\mathrm{D}}=\left\{s: \operatorname{det}\left(s I_{n}-A_{0}-\mathrm{e}^{\left(A_{0}+B K_{0}\right) r} B K_{0} \mathrm{e}^{-s r}\right)=0\right\} .
$$

It follows that we need only to show that $\lambda_{\mathrm{ID}} \cup \lambda\left(A_{0}+B K_{0}\right)=\lambda_{\mathrm{D}}$. Notice that

$$
\begin{aligned}
& \operatorname{det}\left(s I_{n}-\left(A_{0}+B K_{0}\right)\right) \operatorname{det}\left(I_{m}+\int_{-r}^{0} K_{0} \mathrm{e}^{-\left(A_{0}+B K_{0}\right) \theta} B \mathrm{e}^{s \theta} \mathrm{d} \theta\right) \\
= & \operatorname{det}\left(s I_{n}-\left(A_{0}+B K_{0}\right)\right) \operatorname{det}\left(I_{m}+K_{0} \int_{-r}^{0} \mathrm{e}^{\left(s I_{n}-\left(A_{0}+B K_{0}\right)\right) \theta} \mathrm{d} \theta B\right) \\
= & \operatorname{det}\left(s I_{n}-\left(A_{0}+B K_{0}\right)\right) \operatorname{det}\left(I_{m}+K_{0}\left(s I_{n}-\left(A_{0}+B K_{0}\right)\right)^{-1}\left(I_{n}-\mathrm{e}^{-\left(s I_{n}-\left(A_{0}+B K_{0}\right)\right) r}\right) B\right) \\
= & \operatorname{det}\left(s I_{n}-\left(A_{0}+B K_{0}\right)\right) \operatorname{det}\left(I_{n}+\left(I_{n}-\mathrm{e}^{-\left(s I_{n}-\left(A_{0}+B K_{0}\right)\right) r}\right) B K_{0}\left(s I_{n}-\left(A_{0}+B K_{0}\right)\right)^{-1}\right) \\
= & \operatorname{det}\left(\left(I_{n}+\left(I_{n}-\mathrm{e}^{-\left(s I_{n}-\left(A_{0}+B K_{0}\right)\right) r}\right) B K_{0}\left(s I_{n}-\left(A_{0}+B K_{0}\right)\right)^{-1}\right)\left(s I_{n}-\left(A_{0}+B K_{0}\right)\right)\right) \\
= & \operatorname{det}\left(\left(s I_{n}-\left(A_{0}+B K_{0}\right)\right)+\left(I_{n}-\mathrm{e}^{-\left(s I_{n}-\left(A_{0}+B K_{0}\right)\right) r}\right) B K_{0}\right) \\
= & \operatorname{det}\left(s I_{n}-A_{0}-\mathrm{e}^{\left(A_{0}+B K_{0}\right) r} B K_{0} \mathrm{e}^{-s r}\right),
\end{aligned}
$$

where we have used $\operatorname{det}(I+C L)=\operatorname{det}(I+L C)$ with $C$ and $L$ having appropriate dimensions. The proof is thus finished.

Before closing this subsection, we give some remarks as follows.

Remark 2 We will declare that the PPF approach also solves the stabilization problem for retarded-type time-delay systems with both state and input delays. Let $D=0$. Then system (1) becomes the following retarded-type linear time-delay system

$$
\dot{x}(t)=A_{0} x(t)+A_{1} x(t-r)+B u(t-r),
$$

and system (2) becomes

$$
\dot{x}(t)=\left(A_{0}+B K_{0}\right) x(t)+\left(A_{1}+B K_{1}\right) x(t-r) .
$$

The corresponding PPF (10) becomes

$$
u(t)=\left(K_{0} \mathrm{e}^{\left(A_{0}+B K_{0}\right) r}+K_{1}\right) x(t)+K_{0} \int_{t-r}^{t} \mathrm{e}^{\left(A_{0}+B K_{0}\right)(t-s)}\left(A_{1}+B K_{1}\right) x(s) \mathrm{d} s .
$$

Then we have the same results as Theorem 1 and Corollary 1, which will be omitted for brevity.

Remark 3 We mention that the PPF (10) possess several advantages over the conventional predictor feedback (7). On the one hand, it is interesting to note that (12) has the similar structure to (8). However, the stability of the IDS (8) is a necessary condition for the stability of the closed-loop system consisting of (1) and (7), while the stability of the IDS (12) is a necessary and sufficient condition for the stability of the closed-loop system (11). On the other hand, in order to guarantee the stability of IDS (8), the kernel may be needed to be small. Particularly if $A_{0}$ is not Hurwitz, $A_{0}+B K_{0}$ is chosen to be Hurwitz, which contributes to a small kernel. As a consequence, IDS (12) is much more easy to be satisfied than IDS (8). 
Although the PPF (10) also contains memory terms as the conventional predictor feedback (7), in this subsection we will prove that, different from the conventional predictor (7), the PPF controller (10) can be safely implemented without using any input filters. Notice that the PPF controller (10) can be rewritten as

$$
\begin{aligned}
u(t)= & \left(K_{0}\left(\mathrm{e}^{\left(A_{0}+B K_{0}\right) r}+D\right)+K_{1}\right) x(t)-K_{0} \mathrm{e}^{\left(A_{0}+B K_{0}\right) r} D x(t-r) \\
& +K_{0} \int_{0}^{r} \mathrm{e}^{\left(A_{0}+B K_{0}\right) s}\left(\left(A_{0}+B K_{0}\right) D+A_{1}+B K_{1}\right) x(t-s) \mathrm{d} s .
\end{aligned}
$$

Based on some fixed-step numerical integration methods, the distributed (memory) term in the PPF controller (14) can be approximated as

$$
\begin{aligned}
& \int_{0}^{r} \mathrm{e}^{\left(A_{0}+B K_{0}\right) s}\left(\left(A_{0}+B K_{0}\right) D+A_{1}+B K_{1}\right) x(t-s) \mathrm{d} s \\
\approx & \sum_{i=0}^{N} \eta(i) \mathrm{e}^{\left(A_{0}+B K_{0}\right) \frac{i r}{N}}\left(\left(A_{0}+B K_{0}\right) D+A_{1}+B K_{1}\right) x\left(t-\frac{i r}{N}\right),
\end{aligned}
$$

where $N$ is the number of integration step, and $\eta(i)$ are coefficients that depend on the selected integration rule. It then follows from (15) that the PPF controller (14) can be approximated as

$$
\begin{aligned}
u(t)= & \left(K_{0}\left(\mathrm{e}^{\left(A_{0}+B K_{0}\right) r}+D\right)+K_{1}\right) x(t)-K_{0} \mathrm{e}^{\left(A_{0}+B K_{0}\right) r} D x(t-r) \\
& +K_{0} \sum_{i=0}^{N} \eta(i) \mathrm{e}^{\left(A_{0}+B K_{0}\right) \frac{i r}{N}}\left(\left(A_{0}+B K_{0}\right) D+A_{1}+B K_{1}\right) x\left(t-\frac{i r}{N}\right) .
\end{aligned}
$$

In the following proposition, whose proof is provided in Appendix, we show that the stability of the closedloop system can still be maintained provided the above numerical integration is accurate enough.

Proposition 1 Assume that the closed-loop system consisting of (1) and (14) is asymptotically stable. Then there exists a large enough integer $N^{*}>0$ such that the closed-loop system consisting of (1) and (16) remains asymptotically stable for all $N \geq N^{*}$.

\subsection{Computations of the Feedback Gains}

In order to synthesize a stabilizing feedback controller we need to find values for gains $F_{1}$ and $F_{2}$ such that both the zero solution of equation (4) and the zero solution of equation (16) are exponentially stable. To solve this simultaneous stabilization problem we adopt the direct optimization approach for systems described by delay differential algebraic equations (DDAEs), as developed in [20] and the references therein. More precisely we determine the gains by solving the optimization problem

$$
\min _{F_{1}, F_{2}} \alpha\left(F_{1}, F_{2}\right)
$$

where

$$
\alpha\left(F_{1}, F_{2}\right)=\max \left(\alpha_{1}\left(F_{1}, F_{2}\right), \alpha_{2}\left(F_{1}, F 2\right)\right),
$$

with $\alpha_{1}$ and $\alpha_{2}$ being spectral abscissa of (4) and (16),

$$
\begin{aligned}
& \alpha_{1}\left(F_{1}, F_{2}\right):=\sup _{\lambda \in \mathbb{C}}\left\{\Re(\lambda): \operatorname{det}\left(\lambda I-\left(A_{1}+B F_{1}\right)-\left(A_{2}+B F_{2}\right) \mathrm{e}^{-\lambda r}\right)=0\right\}, \\
& \alpha_{2}\left(F_{1}, F_{2}\right):=\sup _{\lambda \in \mathbb{C}}\left\{\Re(\lambda): \operatorname{det}\left(I+\int_{0}^{h}\left(F_{1} K(u)+F_{2} K(u-r)\right) B \mathrm{e}^{-\lambda u} \mathrm{~d} u\right)=0\right\} .
\end{aligned}
$$

The system is stabilizable if the global minimum is strictly negative, and for exponential stability it is sufficient to find gain values resulting in a strictly negative objective function.

Optimization problem (17) is in general non-convex. The objective function may be not everywhere differentiable, due to the maximum operators in (18). At points where a rightmost eigenvalue is multiple and defective, it may even be not locally Lipschitz continuous. These properties preclude the use of standard optimization methods, developed for smooth problems. Instead we use a combination of the Broyden-FletcherGoldfarb-Shanno (BFGS) algorithm with weak Wolfe line search and gradient sampling, as implemented in 
the Matlab code Hybrid Algorithm for Non-smooth Optimaztion (HANSO [24]). This code only requires a routine for the evaluation of the objective function, as well as its derivatives with respect to the controller parameters, whenever it is differentiable. The latter is satisfied if in the closed upper complex plane there is only one rightmost eigenvalue of multiplicity one. The value of the objective function $\alpha$ is obtained by computing the rightmost eigenvalue using a procedure similar as in [?], and the gradient with respect to the controller gains is obtained from the corresponding left and right eigenvectors, see [20] for the details.

Instead of explaining the algorithm in full detail, we highlight a few points where tailoring the approach of [20] to the problem under consideration deserves special attention and necessitates adaptations to the code. First, the software corresponding to [20] has been designed for systems with discrete delays. Therefore, under assumption of commensurate delays, the rightmost eigenvalues of (16) are computed as the rightmost eigenvalues of (??), followed by an automated removal of eigenvalues of matrix $\tilde{A}$, which is in accordance to Proposition ??.

Second, we need to provide a routine for computing the derivative of an eigenvalue of, system (??), with characteristic equation (??) with respect to the elements of $F_{1}$ and $F_{2}$, which we group in a vector $f=$ $\left[\begin{array}{lll}f_{1} & \cdots & f_{p}\end{array}\right]$. Using expression (55) in [20], this involves determining the partial derivatives of matrix

$$
M(f):=\mathrm{e}^{\tilde{A} \delta} \tilde{B}
$$

where $\tilde{A}$ explicitly depends on $f$, while $\tilde{B}$ depends on $\tilde{A}$ via (??). As starting point we express

$$
M(f)=\tilde{K}(\delta ; f)
$$

where function $s \mapsto \tilde{K}(s ; f)$ satisfies boundary value problem (??)-(??). Note here that, whenever needed for clarity, we stress the dependence on parameters $f$ by a second argument separated by a dot-comma. Letting $\tilde{K}_{f_{i}}(s ; f)=\frac{\partial \tilde{K}}{\partial f_{i}}(s ; f)$, we can express

$$
\frac{\partial M}{\partial f_{i}}(f)=\tilde{K}_{f_{i}}(\delta ; f), \quad i=1, \ldots, p .
$$

Taking the derivative of (??)-(??) with respect to parameter $f_{i}$ leads to a new boundary value problem:

$$
\left\{\begin{array}{l}
\dot{\tilde{K}}_{f_{i}}(s ; f)=\tilde{A} \tilde{K}_{f_{i}}(s ; f)+\frac{d \tilde{A}}{d f_{i}} \tilde{K}(s ; f), s \in[0, \delta], \\
\tilde{K}_{f_{i}}(0 ; f)+N \tilde{K}_{f_{i}}(\delta ; f)=0 .
\end{array}\right.
$$

Now, letting

$$
\Xi(s)=\left[\begin{array}{c}
\tilde{K}(s) \\
\tilde{K}_{f_{1}}(s) \\
\vdots \\
\tilde{K}_{f_{p}}(s)
\end{array}\right]
$$

we can compactly rewrite (??)-(??), combined with (21) for $i=1, \ldots, p$, in the following form,

$$
\dot{\Xi}(s)=\left[\begin{array}{cccc}
\tilde{A} & 0 & \cdots & 0 \\
\frac{d \tilde{A}}{d f_{1}} & \tilde{A} & & \\
\vdots & & \ddots & \\
\frac{d \tilde{A}}{d f_{p}} & 0 & \cdots & \tilde{A}
\end{array}\right] \dot{\Xi}(s), \quad s \in[0,1],
$$

supplemented with

$$
\Xi(0)+\left[\begin{array}{cccc}
N & & & \\
& N & & \\
& & \ddots & \\
& & & N
\end{array}\right] \Xi(\delta)=\left[\begin{array}{c}
W \\
0 \\
\vdots \\
0
\end{array}\right]
$$

It is important to note that (22)-(23) has exactly the same structure as (??)-(??). Hence it can be solved in the same way (analytically in terms of matrix exponentials or numerically), allowing to evaluate function $M(f)$ and all its derivatives simultaneously. 


\section{References}

[1] Bellen, A., Guglielmi, N. \& Ruehli, A. E. (1999). Methods for linear systems of circuit delay differential equations of neutral type, IEEE Transactions on Circuits and Systems I: Fundamental Theory and Applications, 46(1), 212-215.

[2] Bekiaris-Liberis, N., \& Krstic, M. (2010). Stabilization of linear strict-feedback systems with delayed integrators, Automatica, 46(11), 1902-1910.

[3] Bekiaris-Liberis, N., \& Krstic, M. (2011). Lyapunov stability of linear predictor feedback for distributed input delays, IEEE Transactions on Automatic Control, 56(3), 655-660.

[4] Cacace, F., \& Germani, A. (2017). Output feedback control of linear systems with input, state and output delays by chains of predictors, Automatica, 85, 455-461.

[5] Chen, W. H., \& Zheng, W. X. (2007). Delay-dependent robust stabilization for uncertain neutral systems with distributed delays, Automatica, 43(1), 95-104.

[6] Fiagbedzi, Y. A. \& Pearson, A. E. (1986). Feedback stabilization of linear autonomous time lag systems, IEEE Transactions on Automatic Control, 31(9), 847-855.

[7] Gantmacher, F. R. (1959). The Theory of Matrix, New York: AMS Chelsea.

[8] Karafyllis, I. (2011). Stabilization by means of approximate predictors for systems with delayed input, SIAM Journal on Control and Optimization, 49(3), 1100-1123.

[9] Kharitonov, V. L. (2014). An extension of the prediction scheme to the case of systems with both input and state delay, Automatica, 50(1), 211-217.

[10] Kharitonov, V. L. (2015). Predictor based stabilization of neutral type systems with input delay, Automatica, 52, 125-134.

[11] Kolmanovskii, V. B., \& Nosov, V. R. (1986). Stability of functional differential equations. London: Academic Press.

[12] Krstic, M. (2010). Lyapunov stability of linear predictor feedback for time-varying input delay. IEEE Transactions on Automatic Control, 55(2), 554-559.

[13] Liu, K. Z., Sun, X. M., \& Krstic, M. (2018). Distributed predictor-based stabilization of continuous interconnected systems with input delays, Automatica, 91, 69-78.

[14] Liu, Q. \& Zhou, B. (2016). Delay compensation of discrete-time linear systems by nested prediction, IET Control Theory \& Applications, 10(15), 1824-1834.

[15] Liu, Q. \& Zhou, B. (2018). Delay compensation for linear systems with both state and distinct input delays, International Journal of Robust and Nonlinear Control, 28, 4455-4478.

[16] Liu, Q. \& Zhou, B. (2018). Regulation of linear systems with both pointwise and distributed input delays by memoryless feedback, to appear.

[17] Murray, R. M., Jacobson, C. A., Casas, R., Khibnik, A. I., Johnson Jr., C. R., Bitmead, R., Peracchio, A. A., \& Proscia, W. M. (1998). System identification for limit cycling systems: A case study for combustion instabilities, In Proceedings of the American Control Conference, 2004-2008, Philadelphia, American.

[18] Michiels, W., \& Vyhlidal, T. (2005). An eigenvalue based approach for the stabilization of linear timedelay systems of neutral type, Automatica, 41(6), 991-998.

[19] Michiels, W., Mondie S. \& Roose, D. (2003). Robust stabilization of time-delay systems with distributed delay control laws: Necessary and sufficient conditions for a safe implementation. Dept. Comput. Sci., Katholieke Univ. Leuven, Leuven, Belgium, Tech. Rep. TW363.

[20] W. Michiels. (2011). Spectrum based stability analysis and stabilization of systems described by delay differential algebraic equations. IET Control Theory and Applications, 5(16), 1829-1842. 
[21] W. Michiels, S. Mondi e, D. Roose, and M. Dambrine. (2004). The effect of approximating distributed delay control laws on stability. In Advances in Time-Delay Systems, Lecture Notes in Computational Science and Engineering, 207-225. Springer Verlag.

[22] Mondie S. \& Michiels, W. (2003). Finite spectrum assignment of unstable time-delay systems with a safe implementation, IEEE Transactions on Automatic Control, 48(12), 2207-2212.

[23] Niculescu, S.-I., \& Brogliato, B. (1999). Fore measurements time-delays and contact instability phenomenon, European Journal of Control, 5, 279-289.

[24] M. Overton. (2009). HANSO: a hybrid algorithm for nonsmooth optimization. Available from http://cs.nyu.edu/overton/software/hanso/.

[25] Richard, J. P. (2003). Time-delay systems: an overview of some recent advances and open problems, Automatica, 39(10), 1667-1694.

[26] Sanz, R., Garcia, P., Fridman, E., \& Albertos, P. (2018). Rejection of mismatched disturbances for systems with input delay via a predictive extended state observer, International Journal of Robust and Nonlinear Control, 28(6), 2457-2467.

[27] Smith, O. J. M. (1959). A controller to overcome dead time, ISA Journal, 6(2), 28-33.

[28] Van Assche, V., Dambrine, M., Lafay, J. F. \& Richard, J. P. (1999). Some problems arising in the implementation of distributed-delay control laws, In 38th IEEE Conference on Decision and Control, 4668-4672.

[29] Watanabe, K. \& Ito, M. (1981). A process-model control for linear systems with delay, IEEE Transactions on Automatic Control, 26, 1261-1269.

[30] Wang, Q. G., Lee, T. H., \& Tan, K. K. (1998). Finite-Spectrum Assignment for Time-Delay Systems. Springer Science \& Business Media.

[31] Wu, Z. \& Michiels, W. (2012). Reliably computing all characteristic roots of delay differential equations in a given right half plane, Journal of Computational and Applied Mathematics, 236, 2499-2514.

[32] Zhang, B., Lam, J. \& Xu, S. (2016). Relaxed results on reachable set estimation of time-delay systems with bounded peak inputs, International Journal of Robust and Nonlinear Control, 26(9), 1994-2007.

[33] Zhou, B. (2014). Pseudo-predictor feedback stabilization of linear systems with time-varying input delays, Automatica, 50(11), 2861-2871.

[34] Zhou, B. (2014). Truncated Predictor Feedback for Time-delay Systems, XIX, 480 p., Heidelberg, Germany: Springer-Verlag.

[35] Zhou, B. \& Liu, Q. (2017). Input delay compensation for neutral type time-delay systems, Automatica, 78, 309-319.

[36] Zhou, B. (2014). Input delay compensation of linear systems with both state and input delays by nested prediction, Automatica, 50(5), 1434-1443. 